www. revis tad y o. com

\title{
Modelo para la Evaluación de la Satisfacción Laboral en una Organi- zación Policial
}

Model for the Evaluation of job satisfaction in a police organization

\author{
Carlos O. Figueroa Pérez ${ }^{1}$, Juan C. Sosa Varela ${ }^{1}$, Jesús Juan Ruiz ${ }^{2}$ y Francisco J. Rivera Pérez ${ }^{1}$ \\ ${ }^{1}$ Escuela de Negocios y Empresarismo, Universidad del Turabo \\ 2 Universidad Politécnica de Madrid \\ carlos.figueroa7@upr.edu, jsosa4@suagm.edu, jjuan@etsii.upm.es, ut_fjrivera@suagm.edu
}

Fecha de recepción: 23-01-2015

Fecha de aceptación: 21-10-2015

Resumen: La satisfacción laboral del policía es una condición imprescindible para un adecuado cumplimiento de las funciones que la sociedad le encomienda. En la satisfacción del policía influyen factores y condiciones del ámbito laboral además de actitudes vitales ajenas a su actividad profesional. Este trabajo aborda por primera vez la satisfacción laboral de la Policía en Puerto Rico. Se provee interés especial por la dificultad de encontrar investigaciones en el marco de organizaciones cuya estructura de relaciones personales se fundamenten en el principio de autoridad. Se han construido varios indicadores de satisfacción basados en las opiniones del propio policía respecto a sus condiciones de trabajo, comunicación entre los distintos niveles de la jerarquía y relaciones personales. A continuación se analizan los condicionantes que influyen en la satisfacción laboral del policía. El resultado del trabajo es un punto de partida para estudiar la evolución de la satisfacción laboral de los policías y establecer políticas de mejoras.

Palabras clave: Satisfacción Laboral, Autoridad y Comunicación, Recursos y Desarrollo y Ambiente Institucional

\begin{abstract}
Police Job satisfaction is a prerequisite for the proper performance of the functions entrusted society. Police satisfaction factors and conditions affecting the workplace well beyond their professional activity vital attitudes. This paper addresses first job satisfaction Police in Puerto Rico. Special interest is provided by the difficulty of finding investigations within organizations whose structure relationships are based on the principle of authority. They have built several indicators of satisfaction based on the views of the police regarding their own working conditions, communication between different levels of the hierarchy and relationships. Here are the factors that influence job satisfaction police are analyzed. The result of this study is a starting point for studying the evolution of job satisfaction of the police and establishes policies for improvement.
\end{abstract}

Keywords: Job Satisfaction, Authority and Communication, Resources and Development and Institutional Environment.

\section{Introducción}

De acuerdo con la literatura analizada existen muchos factores que contribuyen a crear satisfacción e insatisfacción en los empleados de cualquier empresa o institución. Hablando en términos generales, la satisfacción laboral se determina por múltiples factores que están relacionados con la motivación personal, los logros, el nivel de responsabilidad, el reconocimiento, la estructura organizativa, la supervisión, el salario, las condiciones de trabajo, las relaciones entre compañeros y la comunicación con los superiores entre otros. Cuando los empleados están trabajando bajo niveles de insatisfacción laboral, la probabilidad de que disminuya el rendimiento es mayor (Shane, 2008). La satisfacción laboral de un policía en particular incluye elementos diferenciales aún más difíciles de medir, asociados a la institución que incluye el prestigio del organismo y el sentimiento de pertenencia a la organización. Estudios sobre la satisfacción en el trabajo han examinado características demográficas como la raza, la educación y la edad pero investigaciones recientes sugiere que los factores asociados con la satisfacción laboral pueden ser sobre un departamento específico o ambiente organizacional (Zhao, 1999; Buzawa, 1994). El concepto de criminalidad es de enorme complejidad y se encuentra en evolución permanente, lo que obliga a un replanteamiento constante de las organizaciones policiales para garantizar la seguridad de los ciudadanos de un país (Goltz, 2006). Este tipo de organizaciones presenta múltiples peculiaridades que las diferencia de otras organizaciones empresariales y de agencias u organismos gubernamentales más 
tradicionales y flexibles. El número de publicaciones académicas dedicadas a su estudio es escaso y la información disponible sobre su funcionamiento interno es muy reducida, por múltiples razones, entre las que se encuentra la propia defensa de la seguridad ciudadana.

En este trabajo queremos atiborrar estudiando los factores claves que influyen en el desempeño de la actividad de un policía.

Frost (2006) instituye que una fase fundamental para reforzar el compromiso institucional de la policía es conocer diversos factores organizacionales. La diagnosis general sobre la institución es que la actividad propia del policía crea en la organización un ambiente inhóspito y altamente estresante, donde los agentes han de soportar una variedad de problemas cotidianos generados internamente por la propia organización, Shane (2008). Por otra parte (Shane, et al., 2010) identifica como causa fundamental del estrés la disposición estructural de la organización, en la que los agentes ejercen muy poco control (Cherniss, 1980. Para algunos investigadores la satisfacción en el trabajo se convierte en un aspecto esencial del compromiso organizacional (Williams \& Hazzer (1986). Autores como Chiang, Salazar y Núñez (2007) identificaron que la comunicación interna, reconocimiento, relaciones interpersonales, calidad de trabajo, toma de decisiones, objetivos de la institución, compromiso y adaptación al cambio afectan el clima organizacional. Por otra parte Patlán et al (2012) plantean que la satisfacción laboral, tiene impactos significativos en el desempeño, el bienestar en el trabajo y la rotación laboral. La satisfacción laboral, ha sido reconocida en la industria privada como requisito previo para un desempeño laboral exitoso (Griffin, Dunbar y McGrill, 1978). El concepto satisfacción se deriva de las evaluaciones relativas a los aspectos específicos del trabajo, mientras que el compromiso se refiere a las evaluaciones de la organización en su conjunto.

La construcción de la satisfacción en el trabajo y sus consecuencias han sido en gran medida ignorado por la carrera policial (Mire, 2007). Spector (1985) define el concepto de satisfacción laboral como los sentimientos que experimentan los trabajadores respecto a su trabajo y diferentes aspectos de éste. El modelo de Spector (Job Satisfaction Survey, Spector, 1985) se utiliza como referencia por proveer un cuestionario que descompone la satisfacción global en sus componentes básicos.

En la literatura, existe un amplio número de variables y antecedentes relacionados con la satisfacción laboral (Alonso, 2008). Estos factores pueden agruparse en características personales como lo es personalidad, edad, escolaridad, características del puesto, actividad, nivel jerárquico, salario, características de las organización, liderazgo, trabajo en equipo, estructura y apoyo (Patlán, J., Martínez, E,. \& Hernández, R. 2012). Gabriunas (2010) establece que la satisfacción laboral ha sido asociada en la literatura con importantes resultados individuales y organizacionales. A nivel individual, se ha encontrado que la satisfacción afecta actitudes y comportamientos en el trabajo, tales como el compromiso organizacional, el desempeño, el ausentismo y la rotación (Harrison, Newman y Roth, 2006; Judge, Thoresen, Bono y Patton, 2001).

\section{Fundamentos sobre Autoridad y Comunicación}

La autoridad desde la perspectiva policiaca está asociada con la estructura organizacional de los puestos de trabajo. En lo que a la policía se refiere la distribución de puestos refleja una estructura cuasi militar en todos los departamentos, enmarcado por líneas claras de jerarquía.

La autoridad se refleja constantemente en un sistema formal de reglas y regulaciones que gobiernan todas las acciones oficiales (Shane, 2008). El modelo burocrático de la policía tiene tendencia a minar la motivación y la iniciativa de sus empleados. Por otra parte, el avance profesional (es decir, promoción) se basa en la mayoría de los casos en una evaluación externa (examen oficial) no vinculada a su desempeño (Bittner, 1970, 1990; Fogelson, 1977; Kroes, 1985; Manning, 1977).

Esto, según Álvarez (2009) con mucha frecuencia determina aspectos claves de la actividad policial que pueden ser significativa en el éxito de las labores que realiza un policía, como la asignación de funciones, competencias, la dependencia orgánica y funcional, o la simple distribución territorial.

La Policía de Puerto Rico (PPR) es una organización cuasi militar en donde la autoridad conlleva en muchas ocasiones la razón y es determinante en la relación entre un empleado y sus superiores. Este ejercicio de la autoridad formal, promulgado por el taylorismo como método único de coordinación, mientras que en otros ámbitos atraviesa un momento de seria dificultad en las organizaciones modernas (Monsalve, Broncano, \& Niño, 2012), es inherente e incuestionable en una organización policial. El concepto policía representa simbólicamente el orden, la moral y la norma dominante, es la línea divisoria entre "lo bueno y lo malo" definido en una sociedad (Torrente, 1992, 1997). 
La función de la policía se clasifica en tres tipos: (1) aplicar la ley, (2) mantener el orden y (3) prestar servicios a la comunidad (Wilson, 1968). En Puerto Rico, la policía ejerce en muchas localidades otra función más, representar al gobierno en el poder, función que hace a la institución muy vulnerable desde el punto de vista social. La estructura jerárquica y las reglas que rigen las relaciones entre los distintos niveles de dicha estructura se contrapone a los principios aceptados en los que se deben fundamentar el gobierno de una Policía democrática, en un Estado de Derecho que privilegie el respeto a los derechos humanos y las libertades individuales (Miranda, 2009).

Bruce (2003) propone en su "Democratic reform of police - any lessons for Kenya from South Africa", lo que según la experiencia internacional son los aspectos que debe considerar toda reforma policial que busque una orientación democrática: (a) despolitizar el servicio y la administración policiaca, (b) establecer un sistema equitativo en la estructura de rangos $y$, uno de los puntos de mayor relevancia, (c) el desarrollo de la carrera profesional del policía basado en principios de méritos, transparencia y justicia.

El correcto funcionamiento de una organización policial debe cuidar muy especialmente la comunicación entre los distintos niveles. Esta comunicación consiste en la transmisión de información dentro de la organización (Seven, 2012). Este aspecto, ha sido un tema que ha recibido gran atención en los últimos años (Goris, 2000) y ha sido una característica determinante en la evaluación de la satisfacción laboral de los policías.

Uno de los objetivos de este trabajo es establecer la relación de la satisfacción con las variables que caracterizan las relaciones laborales (autoridad) y el flujo de información (comunicación) en la organización policiaca.

\section{Fundamentos sobre Recursos y Desarrollo}

Dentro del apartado de recursos y desarrollo se incluyen las herramientas y equipo del que dispone el policía para el ejercicio de su trabajo y la preparación y formación que le capacitan para dicho ejercicio.

Los recursos y desarrollo en la organización de la PPR están muy asociados a las herramientas disponibles para la prevención del crimen y los medios puestos a su alcance por la agencia para su desarrollo profesional.

La preocupación dentro de este apartado se centra más en la adecuación del equipo que en la formación y adiestramiento de los individuos. La evolución y el proceso de mejora del policía se fundamentan en motivaciones personales, ánimo de superación en un trabajo muy vocacional y en las expectativas profesionales que le brinda la organización. En estos aspectos son interesantes detectar las diferentes percepciones entre generaciones de policías y la virtual competencia que existe entre el policía experimentado y el recién incorporado.

Según (Uluturk 2012) los recursos y desarrollo son importantes en la determinación de la satisfacción laboral. Este énfasis fue recreado por el equipo de William Bratton jefe de la policía de Nueva York en donde se llevó a cabo una encuesta con casi 8,000 agentes de la policía de Nueva York y recibió cerca de 600 recomendaciones (Bratton, 1998). De esta manera, durante el proceso de reingeniería, miembros del NYPD se involucraron en el diagnóstico de los problemas y sugirieron soluciones (Silverman, 1999).

El estudio que aquí se presenta es el primero que se hace en la historia de la Policía de Puerto Rico en el que se tiene en cuenta la opinión de los agentes. El estudio se realiza como uno de los pilares para el diseño de un nuevo modelo de organización.

El aumento de los recursos y medios es fundamental pero insuficiente si no se crean las condiciones necesarias para mejorar el rendimiento de la organización. En situaciones en que los niveles de crimen y desorden son muy altos, la reforma policial corre el riesgo de no ser viable, e incluso de ser descalificada por el público y por la propia organización.

Efecto de distracción en un momento en que se requiere el aumento de las capacidades de la Policía; situaciones que sin duda dificultan la implementación de la reforma (Bayley, 2001).

Para una obtención de recursos competentes y un desarrollo ágil y adecuado a la misión y visión de la policía, la proactividad de la alta dirección es transcendental. Hay que destacar que durante el cambio, si la alta dirección pasa por alto la cultura organizacional, incluyendo sus creencias, valores y actitudes, el cambio deseado se puede demorar o no llegar (Doherty y Horne 2001). También estos autores y otros argumentaron que la implementación exitosa de un cambio requiere anticipar y manejar la resistencia (Uluturk 2012).

En este trabajo se presta especial atención al estudio de la influencia en la satisfacción laboral de los atributos y variables relativas a los medios y recursos de 
la policía por una parte y al desarrollo profesional por otra.

\section{Fundamentos sobre Ambiente Institucional}

La policía como toda organización está estructurada en base a un modelo de operación que incluye la asignación de funciones, y competencias teniendo en cuenta la distribución territorial (Álvarez, 2009). Popovich (1998) establece que una organización policial para el cumplimiento de sus objetivos, debe considerar su rendimiento y basar su gestión en el desempeño. También este autor destaca tres modelos identificados a su vez por (Wilson, 1968) en donde maximiza una diferencia entre: modelo legalista, modelo orientado hacia la dispensación de servicios y modelo de vigilancia.

El modelo legalista se caracteriza por la aplicación estricta de las leyes y reglamentos; el uso limitado del poder discrecional; escasas posibilidades de iniciativa propia del policía; un elevado número de detenciones por delitos menores; importancia evaluativa de las detenciones; un importante control jerárquico y división especializada de funciones (Wilson 1968).

El modelo orientado hacia la dispensación de servicios se caracteriza por la descentralización moderada de autoridad y responsabilidades; mayor importancia del mantenimiento del orden vs. la aplicación de la ley; el principal objetivo del cuerpo es ofrecer un servicio público, se concede importancia a las relaciones con la comunidad y opinión pública; tiene elevados valores inculcados a los agentes; existe conciencia individual de las responsabilidades y espíritu de iniciativa. (Wilson 1968).

El modelo de vigilancia se describe como el tipo tradicional norteamericano. Se caracteriza por poco interés por delitos menores; un amplio poder discrecional; la utilización de técnicas persuasivas; pocas oportunidades para iniciativas e innovaciones; un escaso control del personal por sus superiores; limitada descentralización de autoridad y responsabilidad; hay grandes oportunidades para la corrupción (Álvarez, 2009).

Álvarez (2009) tipifica la conducta de los policías en dos modelos: modelo bombero o cartero, según el autor en el primer caso, es un profesional que solo aparece en situaciones de emergencia mientras que el segundo es una persona a la que ves todos los días convirtiéndose en uno más de la comunidad. Torrente, (1997) identifica una serie de características distintivas de la cultura policial:

(1) un sentido del deber que se traduce en una forma de ver la vida; (2) cinismo acerca del orden existente, (3) pesimismo sobre su mejora; (4) sospecha y actitud defensiva ante potenciales peligros; (5) aislamiento de los civiles y solidaridad grupal; (6) conservadurismo moral y social; (7) machismo; (8) prejuicios raciales y pragmatismo".

En el caso de Puerto Rico, la policía está organizada en un sistema de rangos muy amplio y su ambiente organizacional está caracterizado por una continua búsqueda del reconocimiento del trabajo realizado (Meléndez, 2013). Son muchos y variados los estudios académicos relacionados con la cultura organizacional de la policía, la mayoría de estos, aunque expresado de forma distinta, comparte en el fondo ideas comunes que diferencia esta institución de cualquier otra organización empresarial o pública. A partir del marco teórico el objetivo es determinar empíricamente la importancia de cada uno de los atributos establecidos en la satisfacción laboral de la policía. Los resultados de las valoraciones de satisfacción laboral podrían encaminar pautas para mejorar las condiciones laborales, la administración progresiva de los recursos humanos y la calidad de los servicios ofrecidos por la Policía de Puerto por lo que se plantea.

\section{Objetivos de la Investigación}

El objetivo general de la investigación es describir indicadores de satisfacción laboral de los miembros de la PPR, respecto a diversos temas laborales.

Estos indicadores son los puntos de partida para desarrollar planes operacionales y estratégicos enfocados en maximizar los recursos humanos. Un objetivo medular es conocer los índices de satisfacción laboral para reformar los servicios ofertados por parte de la policía, disminuir la burocracia organizacional, y a su vez mejorar el desempeño, reduciendo la comisión de delitos a través de la maximización de los recursos. El trabajo tiene como objetivos identificar el nivel de insatisfacción y sus causas. El proceso ha permitido poner a la organización a trabajar en una dirección, con información objetiva de todos los miembros de la institución.

\section{Modelo de Investigación}

Este estudio está basado en el análisis de los resultados de una encuesta realizada a la Policía de Puerto Rico. En el cuestionario se incluyeron preguntas relati- 
vas a los tres bloques descritos en la sección anterior: (1) Autoridad y Comunicación, (2) Recursos y Desarrollo y (3) Ambiente Institucional. Dentro de cada uno de los bloques se construyó un indicador basado en la técnica de componentes principales. En la primera fase mediante la técnica de componentes principales se obtiene un indicador que determine la satisfacción global del policía. Se ha elegido componentes principales dentro de las opciones de análisis factorial, porque es la técnica que permite reconstruir la información original con mayor precisión a partir de un único factor. Posteriormente, se combinaron los tres indicadores para obtener el indicador global de satisfacción. La Figura 1: muestra el modelo de investigación.

\section{Autoridad y Comunicación}

Recursos y Desarrollo

Ambiente Institucional
Satisfacción Laboral

Fase 1: Componentes Principales

Fuente: Elaboración propia.

Con el propósito de lograr la validez y confiabilidad del instrumento, se utilizó el método de panel de expertos para la redacción del cuestionario. El instrumento fue distribuido en tres ocasiones a un panel de siete especialistas en organizaciones policiales. El cuestionario contiene 60 preguntas en total pero para las áreas identificadas como: Autoridad y Comunicación, Recursos y Desarrollo y Ambiente Institucional se analizaron 29. Las preguntas se diseñaron con una escala de valores que se distribuye entre muy satisfecho (valor 4) hasta muy insatisfecho (valor 1).

La muestra seleccionada que completó la encuesta fue de 448 miembros del Departamento de Policía de Puerto Rico.

\section{Autoridad y Comunicación}

Para evaluar el área de la autoridad y comunicación en el cuerpo policiaco, se utilizaron las preguntas desde la 1.1(a) a la 1.1 (g), (Tabla 1).

Lo que se pretende con estas preguntas es evaluar la satisfacción del cuerpo policiaco con la jerarquía de la organización policiaca y la efectividad en el área de comunicación.
En la tabla 1 se proporciona los valores medios correspondientes a las variables en el apartado de Autoridad y Comunicación. Se observa también la desviación típica de la respuesta (columna SD) que la variabilidad en las respuestas es similar en todas las preguntas.

Como se observa en la tabla 1 y figura 1 , la satisfacción promedio en lo que respecta al área de autoridad y comunicación fue de 2.5. Se observa que las áreas de mayor fortaleza son la comunicación con los compañeros 1.1 (d), con el supervisor inmediato 1.1 (e) y con el subalterno1.1 (g). El grado de satisfacción es menor en las preguntas que involucran a la administración y a la policía como institución. En lo que respecta a la transparencia de la misión y visión de la Institución 1.1(a), es la puntuación más baja en este área. Es razonable que estos valores se encuentren alrededor de los niveles medios y con relativa poca discrepancia entre ellos. Para el tamaño muestra utilizado (333 individuos que respondieron a estas preguntas) las diferencias entre medias superior a 0.07 indican diferencias significativas en su apreciación de la variable. El número de respuestas en este apartado es de 333 en lugar de 420 debido a que muchos policías no tienen subordinados (personas a quienes supervisan) y no contestaron a las preguntas que tenían que ver con ese aspectos. 
Tabla 1. Valor Medio, Desviación Estándar y Número de Respuestas Autoridad y Comunicación

\begin{tabular}{|l|l|l|l|}
\hline & \multicolumn{1}{|c|}{ Mean } & SD & N \\
\hline Misión y Visión 1.1(a) & $\underline{2,37}$ &, 891 & 333 \\
\hline Comunicación con mayor Jerarquía 1.1 (b) & 2,57 &, 894 & 333 \\
\hline Comunicación Administración 1.1 (c) & 2,48 &, 794 & 333 \\
\hline Comunicación Compañeros 1.1 (d) & $\underline{3,24}$ &, 753 & 333 \\
\hline Comunicación Sup. Inmediato 1.1 (e) & $\underline{3,11}$ &, 891 & 333 \\
\hline Capacidad Decisión Sup. 1.1 (f) & 2,80 &, 858 & 333 \\
\hline Comunicación Subalterno 1.1 (g) & 3,05 &, 699 & 333 \\
\hline
\end{tabular}

Tabla 2. Matriz de correlaciones de Autoridad y Comunicación

\begin{tabular}{|c|c|c|c|c|c|c|c|c|}
\hline & & $\begin{array}{l}\text { M i s i ó } n \\
\text { y Visión } \\
1.1(a)\end{array}$ & $\begin{array}{l}\text { Com u n i- } \\
\text { cación con } \\
\text { mayor Jer- } \\
\text { arquía } 1.1 \\
\text { (B) }\end{array}$ & $\begin{array}{l}\text { Comuni- } \\
\text { c a c ión } \\
\text { Adminis - } \\
\text { tración } 1.1 \\
\text { (c) }\end{array}$ & $\begin{array}{l}\text { Comuni- } \\
\text { c a c ión } \\
\text { com- } \\
\text { pañeros } \\
1.1 \text { (d) }\end{array}$ & $\begin{array}{l}\text { Comuni- } \\
\text { c a c i ó n } \\
\text { Sup. Inme- } \\
\text { diato } 1.1(e)\end{array}$ & $\begin{array}{l}\text { Capacidad } \\
\text { Decisión } \\
\text { Sup. } 1.1 \text { (f) }\end{array}$ & $\begin{array}{l}\text { Com uni- } \\
\text { c a c i ó n } \\
\text { Subalterno } \\
1.1 \text { (g) }\end{array}$ \\
\hline \multirow[t]{3}{*}{$\begin{array}{l}\text { M i s i ó n } \\
\text { y Visión } \\
1.1(a)\end{array}$} & $\begin{array}{l}\text { Correlación } \\
\text { de Pearson }\end{array}$ & 1 &, $463\left({ }^{* \star}\right)$ &, $509\left({ }^{* *}\right)$ &, $171\left({ }^{* *}\right)$ &, $188\left(^{* *}\right)$ &, $259\left({ }^{* *}\right)$ &, $\left.293^{(* *}\right)$ \\
\hline & $\begin{array}{l}\text { Sig. (bilat- } \\
\text { eral) }\end{array}$ & &, 000 &, 000 & ,000 & ,000 & ,000 & ,000 \\
\hline & $\mathrm{N}$ & 446 & 439 & 441 & 440 & 444 & 442 & 343 \\
\hline \multirow[t]{3}{*}{$\begin{array}{l}\text { Com u n i- } \\
\text { cación con } \\
\text { mayor Jer- } \\
\text { arquía } 1.1 \\
\text { (B) } \\
\end{array}$} & $\begin{array}{l}\text { Correlación } \\
\text { de Pearson }\end{array}$ & & 1 &, $642\left({ }^{* *}\right)$ &, $235\left(^{* \star}\right)$ &, $295\left(^{* *}\right)$ &, $336\left({ }^{* *}\right)$ &, $378\left({ }^{* *}\right)$ \\
\hline & $\begin{array}{l}\text { Sig. (bilat- } \\
\text { eral) }\end{array}$ & & & ,000 & ,000 &, 000 & ,000 & ,000 \\
\hline & $\mathrm{N}$ & & 441 & 437 & 435 & 439 & 439 & 339 \\
\hline \multirow[t]{2}{*}{$\begin{array}{l}\text { Co m u n i - } \\
\text { cación Ad- } \\
\text { ministración } \\
1.1 \text { (c) }\end{array}$} & $\begin{array}{l}\text { Correlación } \\
\text { de Pearson }\end{array}$ & & & 1 &, $197\left({ }^{* *}\right)$ &, $237\left({ }^{* *}\right)$ &, $308\left({ }^{* *}\right)$ &, $247\left(^{* \star}\right)$ \\
\hline & $\begin{array}{l}\text { Sig. (bilat- } \\
\text { eral) }\end{array}$ & & & & ,000 &, 000 & ,000 &, 000 \\
\hline
\end{tabular}




\begin{tabular}{|c|c|c|c|c|c|c|c|c|}
\hline & & $\begin{array}{l}M \text { i s i ó } n \\
\text { y Visión } \\
1.1(a)\end{array}$ & $\begin{array}{l}\text { Com u n i- } \\
\text { cación con } \\
\text { mayor Jer- } \\
\text { arquía } 1.1 \\
\text { (B) }\end{array}$ & $\begin{array}{l}\text { Comuni- } \\
\text { c a c i ó n } \\
\text { Adminis - } \\
\text { tración } 1.1 \\
\text { (c) }\end{array}$ & $\begin{array}{l}\text { Comuni- } \\
\text { c a c i ón } \\
\text { Co } 0 \text { m- } \\
\text { pañeros } \\
1.1 \text { (d) }\end{array}$ & $\begin{array}{l}\text { Com uni- } \\
\text { c a c i ó n } \\
\text { Sup. Inme- } \\
\text { diato } 1.1(e)\end{array}$ & $\begin{array}{l}\text { Capacidad } \\
\text { Decisión } \\
\text { Sup. } 1.1 \text { (f) }\end{array}$ & $\begin{array}{l}\text { Comuni- } \\
\text { c a c i ó n } \\
\text { Subalterno } \\
1.1(g)\end{array}$ \\
\hline & $\mathrm{N}$ & & & 443 & 437 & 441 & 440 & 343 \\
\hline \multirow{3}{*}{$\begin{array}{l}\text { C o m u - } \\
\text { n ic a ción } \\
\text { C o m - } \\
\text { pañeros } 1.1 \\
\text { (d) }\end{array}$} & $\begin{array}{l}\text { Correlación } \\
\text { de Pearson }\end{array}$ & & & & 1 &, $514\left({ }^{* *}\right)$ &, $322\left({ }^{* *}\right)$ &, $427\left(^{\star \star}\right)$ \\
\hline & $\begin{array}{l}\text { Sig. (bilat- } \\
\text { eral) }\end{array}$ & & & & &, 000 & ,000 & ,000 \\
\hline & $\mathrm{N}$ & & & & 442 & 442 & 439 & 342 \\
\hline \multirow{3}{*}{$\begin{array}{l}\text { Com un i- } \\
\text { cación Sup. } \\
\text { Inmediato } \\
1.1(\mathrm{e})\end{array}$} & $\begin{array}{l}\text { Correlación } \\
\text { de Pearson }\end{array}$ & & & & & 1 &, $607\left(^{* *}\right)$ &, $481\left({ }^{* *}\right)$ \\
\hline & $\begin{array}{l}\text { Sig. (bilat- } \\
\text { eral) }\end{array}$ & & & & & & ,000 & ,000 \\
\hline & $\mathrm{N}$ & & & & & 446 & 443 & 343 \\
\hline \multirow{3}{*}{$\begin{array}{l}\text { Capacidad } \\
\text { De cis ió n } \\
\text { Sup. } 1.1 \text { (f) }\end{array}$} & $\begin{array}{l}\text { Correlación } \\
\text { de Pearson }\end{array}$ & & & & & & 1 &, $509\left({ }^{* *}\right)$ \\
\hline & $\begin{array}{l}\text { Sig. (bilat- } \\
\text { eral) }\end{array}$ & & & & & & & ,000 \\
\hline & $\mathrm{N}$ & & & & & & 444 & 342 \\
\hline \multirow[t]{3}{*}{$\begin{array}{l}\text { Co m u n i - } \\
\text { cación Sub- } \\
\text { alterno } 1.1 \\
\text { (g) }\end{array}$} & $\begin{array}{l}\text { Correlación } \\
\text { de Pearson }\end{array}$ & & & & & & & 1 \\
\hline & $\begin{array}{l}\text { Sig. } \quad \text { (bilat- } \\
\text { eral) }\end{array}$ & & & & & & & \\
\hline & $\mathrm{N}$ & & & & & & & 345 \\
\hline
\end{tabular}


Tabla 3. Componentes principales

Total Variance Explained

\begin{tabular}{|l|r|r|r|r|r|r|}
\hline \multirow{2}{*}{ Component } & \multicolumn{3}{|c|}{ Initial Eigenvalues } & \multicolumn{3}{c|}{ Extraction Sums of Squared Loadings } \\
\cline { 2 - 7 } & \multicolumn{1}{|c|}{ Total } & \% of Variance & Cumulative $\%$ & Total & $\%$ of Variance & Cumulative \% \\
\hline 1 & 3,346 & 47,796 & 47,796 & 3,346 & 47,796 & 47,796 \\
2 & 1,222 & 17,454 & 65,250 & 1,222 & 17,454 & 65,250 \\
3 &, 693 & 9,895 & 75,146 & & & \\
4 &, 569 & 8,122 & 83,268 & & & \\
5 &, 503 & 7,180 & 90,448 & & & \\
6 &, 352 & 5,032 & 95,480 & & & \\
7 &, 316 & 4,520 & 100,000 & & & \\
\hline
\end{tabular}

Extraction Method: Principal Component Analysis.

Tabla 4. Pesos de los componentes principales (Autoridad y Comunicación)

Component Matrix

\begin{tabular}{|c|c|c|}
\hline & \multicolumn{2}{|c|}{ Component } \\
\hline & 1 & 2 \\
\hline Misión y Visión 1.1(a) & ,656 &,- 460 \\
\hline $\begin{array}{l}\text { Comunicación con } \\
\text { may or Jerarquía } 1.1 \text { (B) }\end{array}$ & ,746 &,- 417 \\
\hline $\begin{array}{l}\text { Comunicación } \\
\text { Administración } 1.1 \text { (c) }\end{array}$ & ,692 &,- 537 \\
\hline $\begin{array}{l}\text { Comunicación } \\
\text { Compañeros } 1.1 \text { (d) }\end{array}$ & ,604 & ,395 \\
\hline $\begin{array}{l}\text { Comunicación Sup. } \\
\text { Inmediato } 1.1(\mathrm{e})\end{array}$ & ,712 & ,467 \\
\hline $\begin{array}{l}\text { Capacidad Decisión } \\
\text { Sup. } 1.1(\mathrm{f})\end{array}$ & ,730 & ,252 \\
\hline $\begin{array}{l}\text { Comunicación } \\
\text { Subalterno } 1.1 \text { (g) }\end{array}$ & ,690 & ,332 \\
\hline
\end{tabular}

Extraction Method: Principal Component Analysis.

a. 2 components extracted.

\section{Análisis de Componentes Prin- cipales para Autoridad y Co- municación}

En la tabla 2 se muestra la matriz de correlaciones de las siete variables agrupadas dentro de Autoridad y Comunicación. Se observa que:

a) Las correlaciones son altas y positivas entre todas las variables

b) Todas son significativas con pvalor .000 (muy inferior al límite habitualmente utilizado de 0.05 )

c) Los datos reúnen las condiciones necesarias para que el análisis de componentes principales sea útil. El contraste de Kaiser-Meyer-Olkin proporciona un valor igual a 0.794 y la prueba de esfericidad de
Barlett es muy significativo con un estadístico igual a 791.2 y $\mathrm{p}$ valor muy inferior a los niveles habituales de significación.

d) La conclusión de este bloque de preguntas es clara, existe una percepción de insatisfacción importante que corresponde con las variables que tienen que ver con la comunicación con la institución policial externa a la propia unidad. Si se desea mejorar este aspecto, la solución es simple y está en manos de la administración general de la policía que debe remediar los problemas de comunicación con sus unidades.

e) La tabla 3 muestra los valores propios asociados a cada componente en el análisis de Componentes Principales. Se aprecia que hay una combinación lineal dominante, con un valor propio de 3.34 que representa casi el $50 \%$ de la variabilidad total. En este compo- 
nente todas las variables tienen un peso positivo y muy parecidos. Las dos variables más importantes según la tabla 4 son : comunicación con mayor jerarquía (b) y capacidad de decisión del superior (f). La de menor peso es la Comunicación con Compañeros (d).

Utilizaremos este componente para medir la satisfacción de los policías en relación a este bloque de Autoridad y Comunicación.

f) El segundo componente (tabla 4) muestra el contraste en la satisfacción de los policías entre dos grupos de variables $(a, b$ y $c)$ por un lado y (d, e, f y g) por otro. A igualdad de nivel de satisfacción, los policías que contestan con un valor alto al grupo primero de preguntas ( $a, b$ y $c)$ responden con un valor bajo de satisfacción al segundo grupo (d, e, f y g) y viceversa. La importancia de este segundo componente, aunque inferior al primero, es alta. Sería interesante identificar la causa de esta polarización.

\section{Recursos y Desarrollo}

Para evaluar el área de recursos y desarrollo en el cuer- po policiaco, se utilizaron las preguntas desde la 1.2(a) hasta la $1.2(\mathrm{k})$ del cuestionario. Lo que se pretendía con estas preguntas era evaluar de que forma el cuerpo policiaco estaba satisfecho con los recursos que dispone la Institución y el desarrollo profesional que promueven en la organización. La tasa de respuesta en esta área fue de un $94 \%$. Estas preguntas se diseñaron con una escala de valores que se distribuye entre muy satisfecho (valor 4) hasta muy insatisfecho (valor 1).

Como se observa en la tabla 5, la satisfacción promedio en lo que respecta al área de disponibilidad de equipos fue de 1,68. Esto demuestra que la mayoría de los encuestados están insatisfechos. En particular, su mayor insatisfacción corresponde a las áreas de los recursos financieros correspondientes a salarios, compensaciones, etc. que recibe el cuerpo policial.

Estos dos renglones son de vital importancia para que este cuerpo cumpla con la misión y visión de la organización y que a su vez se perfilan como componentes esenciales para lograr objetivos y metas policiales.

Tabla 5. Valor Medio, Desviación Estándar y Número de Respuestas (Recursos y Desarrollo)

\begin{tabular}{|l|l|l|l|}
\hline & \multicolumn{1}{|c|}{ Mean } & \multicolumn{1}{|c|}{ SD } & N \\
\hline Recursos Tecnológicos 1.2 (a) & 2,01 &, 785 & 420 \\
\hline Recursos Mejoramiento Profesional 1.2 (b) & 1,95 &, 706 & 420 \\
\hline Recursos Educación Continua 1.2 (c) & 2,01 &, 739 & 420 \\
\hline Recursos Financieros 1.2 (d) & 1,45 &, 622 & 420 \\
\hline Equipo Disponible 1.2 (e) & 1,68 &, 711 & 420 \\
\hline La Inmediatez de Recursos 1.2 (f) & 1,90 &, 738 & 420 \\
\hline Oportunidad Desarrollo Profesional 1.2 (g) & 1,78 &, 702 & 420 \\
\hline Reconocimiento Logros 1.2 (h) & 1,98 &, 820 & 420 \\
\hline Oportunidad Ideas 1.2 (i) & 2,04 &, 743 & 420 \\
\hline Proceso de Planificación 1.2 (k) & 1,84 &, 744 & 420 \\
\hline
\end{tabular}


Tabla 6. Matriz de Correlaciones (Recursos y Desarrollo)

\begin{tabular}{|c|c|c|c|c|c|c|c|c|c|c|c|c|}
\hline & & $\begin{array}{l}R \text { e - } \\
\text { cursos } \\
\text { T e c - } \\
\text { nológi- } \\
\text { cos } 1.2 \\
\text { (a) }\end{array}$ & $\begin{array}{l}\text { Recursos } \\
\text { Mejora- } \\
\text { m i e n to } \\
\text { Profesio- } \\
\text { nal } 1.2 \text { (b) }\end{array}$ & $\begin{array}{l}\text { Recursos } \\
\text { Educación } \\
\text { Continua } \\
1.2 \text { (c) }\end{array}$ & $\begin{array}{l}\text { Recursos } \\
\text { Financie- } \\
\text { ros } 1.2 \text { (d) }\end{array}$ & $\begin{array}{l}\text { E q u i p o } \\
\text { Disponible } \\
1.2(\mathrm{e})\end{array}$ & $\begin{array}{l}\text { La Inme- } \\
\text { diatez de } \\
\text { Recur- } \\
\text { sos } 1.2 \\
\text { (f) }\end{array}$ & $\begin{array}{l}\text { Oportuni- } \\
\text { dad Desa- } \\
\text { rrollo Pro- } \\
\text { fesional } \\
1.2 \text { (g) }\end{array}$ & $\begin{array}{l}\text { Recono- } \\
\text { cimiento } \\
\text { Logros } 1.2 \\
\text { (h) }\end{array}$ & $\begin{array}{l}\text { Oportuni- } \\
\text { dad Ideas } \\
1.2 \text { (i) }\end{array}$ & $\begin{array}{l}\text { Acceso } \\
\text { Estudios } \\
\text { Univer- } \\
\text { sitarios } \\
1.2(j)\end{array}$ & $\begin{array}{l}\text { Proceso } \\
\text { de Pla- } \\
\text { nificación } \\
1.2(k)\end{array}$ \\
\hline \multirow[t]{3}{*}{$\begin{array}{l}\text { Recursos } \\
\text { Tecnológi- } \\
\cos 1.2 \text { (a) }\end{array}$} & $\begin{array}{l}\text { Correl- } \\
\text { a ción } \\
\text { d e } \\
\text { Pear- } \\
\text { son }\end{array}$ & 1 &, $\left.650{ }^{* *}\right)$ &, $\left.535^{(* *}\right)$ &, $\left.4399^{* *}\right)$ &, $475\left(^{(*}\right)$ &, $\left.509{ }^{* *}\right)$ &, $\left.432{ }^{* \star}\right)$ &, $\left.363^{(* *}\right)$ &, $\left.430{ }^{* *}\right)$ &, $319\left(^{* *}\right)$ &, $\left.4511^{* *}\right)$ \\
\hline & $\begin{array}{l}\text { Sig. (bi- } \\
\text { lateral) }\end{array}$ & & ,000 & ,000 & ,000 & ,000 & ,000 & ,000 & ,000 & ,000 & ,000 & ,000 \\
\hline & $\mathrm{N}$ & 444 & 441 & 441 & 442 & 444 & 441 & 442 & 439 & 435 & 406 & 433 \\
\hline \multirow[t]{3}{*}{$\begin{array}{l}\text { Recursos } \\
\text { Mejorami- } \\
\text { ento Profe- } \\
\text { sional } 1.2 \\
\text { (b) }\end{array}$} & $\begin{array}{l}\text { Correl- } \\
\text { a ción } \\
\text { d e } \\
\text { P ear - } \\
\text { son }\end{array}$ & & 1 &, $647\left(^{* \star}\right)$ &, $437\left(^{* *}\right)$ &, $489\left(^{* *}\right)$ &, $549\left(^{* *}\right)$ &, $527\left(^{* \star}\right)$ &, $427\left(^{\star *}\right)$ &, $\left.460{ }^{* *}\right)$ &, $412\left(^{* *}\right)$ &, $\left.530{ }^{* *}\right)$ \\
\hline & $\begin{array}{l}\text { Sig. (bi- } \\
\text { lateral) }\end{array}$ & & & ,000 & ,000 & ,000 & ,000 & ,000 & ,000 & ,000 & ,000 & ,000 \\
\hline & $\mathrm{N}$ & & 443 & 441 & 441 & 443 & 440 & 441 & 438 & 434 & 407 & 433 \\
\hline \multirow[t]{3}{*}{$\begin{array}{l}\text { Recursos } \\
\text { Educación } \\
\text { Continua } \\
1.2 \text { (c) }\end{array}$} & $\begin{array}{l}\text { Correl- } \\
\text { ación } \\
\text { d e } \\
\text { Pear- } \\
\text { son }\end{array}$ & & & 1 &, $441\left(^{* *}\right)$ &, $\left.440{ }^{* *}\right)$ &, $\left.604{ }^{* \star}\right)$ &, $507\left(^{\star \star}\right)$ &, $\left.4411^{\star *}\right)$ &, $\left.501{ }^{(*}\right)$ &, $\left.390{ }^{* *}\right)$ &, $523\left(^{* *}\right)$ \\
\hline & $\begin{array}{l}\text { Sig. (bi- } \\
\text { lateral) }\end{array}$ & & & & ,000 & ,000 & ,000 & ,000 & ,000 & ,000 & ,000 & ,000 \\
\hline & $\mathrm{N}$ & & & 443 & 441 & 443 & 440 & 441 & 438 & 435 & 407 & 433 \\
\hline \multirow[t]{3}{*}{$\begin{array}{l}\text { Recursos } \\
\text { Financier- } \\
\text { os } 1.2 \text { (d) }\end{array}$} & $\begin{array}{l}\text { Correl- } \\
\text { a ción } \\
\text { d e } \\
\text { Pear - } \\
\text { son }\end{array}$ & & & & 1 &, $577\left(^{* \star}\right)$ &, $521\left(^{\star \star}\right)$ &, $\left.498{ }^{(* *}\right)$ &, $444\left(^{* *}\right)$ &, $418\left(^{* *}\right)$ &, $317\left(^{\star \star}\right)$ &, $\left.539{ }^{(* *}\right)$ \\
\hline & $\begin{array}{l}\text { Sig. (bi- } \\
\text { lateral) }\end{array}$ & & & & & ,000 & ,000 & ,000 & ,000 & ,000 & ,000 & ,000 \\
\hline & $\mathrm{N}$ & & & & 444 & 444 & 442 & 442 & 439 & 436 & 407 & 433 \\
\hline
\end{tabular}




\begin{tabular}{|c|c|c|c|c|c|c|c|c|c|c|c|c|}
\hline & & $\begin{array}{l}\mathrm{R} \text { e - } \\
\text { cursos } \\
\text { T e c - } \\
\text { nológi- } \\
\text { cos } 1.2 \\
\text { (a) }\end{array}$ & $\begin{array}{l}\text { Recursos } \\
\text { Mejora- } \\
\text { mi en to } \\
\text { Profesio- } \\
\text { nal } 1.2 \text { (b) }\end{array}$ & $\begin{array}{l}\text { Recursos } \\
\text { Educación } \\
\text { Continua } \\
1.2 \text { (c) }\end{array}$ & $\begin{array}{l}\text { Recursos } \\
\text { Financie- } \\
\text { ros } 1.2 \text { (d) }\end{array}$ & $\begin{array}{l}\text { E q u i p o } \\
\text { Disponible } \\
1.2(\mathrm{e})\end{array}$ & $\begin{array}{l}\text { La Inme- } \\
\text { diatez de } \\
\text { Recur- } \\
\text { sos } 1.2 \\
\text { (f) }\end{array}$ & $\begin{array}{l}\text { Oportuni- } \\
\text { dad Desa- } \\
\text { rrollo Pro- } \\
\text { fesional } \\
1.2 \text { (g) }\end{array}$ & $\begin{array}{l}\text { Recono- } \\
\text { cimiento } \\
\text { Logros } 1.2 \\
\text { (h) }\end{array}$ & $\begin{array}{l}\text { Oportuni- } \\
\text { dad Ideas } \\
1.2 \text { (i) }\end{array}$ & $\begin{array}{l}\text { Acceso } \\
\text { Estudios } \\
\text { Univer- } \\
\text { sitarios } \\
1.2(j)\end{array}$ & $\begin{array}{l}\text { Proceso } \\
\text { de Pla- } \\
\text { nificación } \\
1.2 \text { (k) }\end{array}$ \\
\hline \multirow[t]{3}{*}{$\begin{array}{l}\text { E q u i p o } \\
\text { Disponible } \\
1.2(\mathrm{e})\end{array}$} & $\begin{array}{l}\text { Correl- } \\
\text { a ción } \\
d \quad \text { e } \\
\text { Pear- } \\
\text { son }\end{array}$ & & & & & 1 &, $529\left({ }^{\star \star}\right)$ &, $503\left({ }^{\star \star}\right)$ &, $424\left(^{\star \star *}\right)$ &, $\left.405^{(* *}\right)$ &, $\left.2822^{* *}\right)$ &, $540\left(^{* *}\right)$ \\
\hline & $\begin{array}{l}\text { Sig. (bi- } \\
\text { lateral) }\end{array}$ & & & & & & ,000 & ,000 & ,000 & ,000 & ,000 & ,000 \\
\hline & $\mathrm{N}$ & & & & & 447 & 444 & 445 & 442 & 438 & 409 & 436 \\
\hline \multirow[t]{3}{*}{$\begin{array}{l}\text { La Inme- } \\
\text { diatez de } \\
\text { Recursos } \\
1.2 \text { (f) }\end{array}$} & $\begin{array}{l}\text { Correl- } \\
\text { a ción } \\
\text { d e } \\
\text { Pear- } \\
\text { son }\end{array}$ & & & & & & 1 &, $\left.5611^{* \star}\right)$ &, $\left.472{ }^{(* *}\right)$ &, $5322^{(* *)}$ &, $358\left(^{* \star}\right)$ &, $\left.5511^{* *}\right)$ \\
\hline & $\begin{array}{l}\text { Sig. (bi- } \\
\text { lateral) }\end{array}$ & & & & & & & ,000 & ,000 & ,000 & ,000 & ,000 \\
\hline & $\mathrm{N}$ & & & & & & 444 & 442 & 439 & 437 & 409 & 434 \\
\hline \multirow[t]{3}{*}{$\begin{array}{l}\text { Oportuni- } \\
\text { dad Desar- } \\
\text { rollo Profe- } \\
\text { sional } 1.2 \\
\text { (g) }\end{array}$} & $\begin{array}{l}\text { Correl- } \\
\text { ación } \\
\text { d e } \\
\text { Pear- } \\
\text { son }\end{array}$ & & & & & & & 1 &, $\left.529{ }^{* \star}\right)$ &, $\left.5355^{* \star}\right)$ &, $\left.489{ }^{* *}\right)$ &, $569\left(^{* *}\right)$ \\
\hline & $\begin{array}{l}\text { Sig. (bi- } \\
\text { lateral) }\end{array}$ & & & & & & & & ,000 & ,000 & ,000 & ,000 \\
\hline & $\mathrm{N}$ & & & & & & & 445 & 442 & 438 & 409 & 434 \\
\hline \multirow[t]{3}{*}{$\begin{array}{l}\text { Recono- } \\
\text { cimiento } \\
\text { Logros } 1.2 \\
\text { (h) }\end{array}$} & $\begin{array}{l}\text { Correl- } \\
\text { ación } \\
\text { d e } \\
\text { Pear- } \\
\text { son }\end{array}$ & & & & & & & & 1 &, $\left.551^{(* *}\right)$ &, $\left.470{ }^{(* *}\right)$ &, $515\left(^{(* *}\right)$ \\
\hline & $\begin{array}{l}\text { Sig. (bi- } \\
\text { lateral) }\end{array}$ & & & & & & & & & ,000 & ,000 & ,000 \\
\hline & $\mathrm{N}$ & & & & & & & & 443 & 436 & 406 & 433 \\
\hline $\begin{array}{l}\text { Oportund- } \\
\text { dad Ideas } \\
1.2 \text { (i) }\end{array}$ & $\begin{array}{l}\text { Correl- } \\
\text { ación } \\
\text { d e } \\
\text { Pear- } \\
\text { son }\end{array}$ & & & & & & & & & 1 &, $\left.4644^{(\star *}\right)$ &, $555\left({ }^{(* *}\right)$ \\
\hline
\end{tabular}




\begin{tabular}{|c|c|c|c|c|c|c|c|c|c|c|c|c|}
\hline & & $\begin{array}{l}R \text { R e - } \\
\text { cursos } \\
\text { T e c }- \\
\text { nológi- } \\
\text { cos } 1.2 \\
\text { (a) }\end{array}$ & $\begin{array}{l}\text { Recursos } \\
\text { Mejora- } \\
\text { miento } \\
\text { Profesio- } \\
\text { nal } 1.2 \text { (b) }\end{array}$ & $\begin{array}{l}\text { Recursos } \\
\text { Educación } \\
\text { Continua } \\
1.2 \text { (c) }\end{array}$ & $\begin{array}{l}\text { Recursos } \\
\text { Financie- } \\
\text { ros } 1.2 \text { (d) }\end{array}$ & $\begin{array}{l}\text { E q u i p o } \\
\text { Disponible } \\
1.2 \text { (e) }\end{array}$ & $\begin{array}{l}\text { La Inme- } \\
\text { diatez de } \\
\text { Recur- } \\
\text { sos } 1.2 \\
\text { (f) }\end{array}$ & $\begin{array}{l}\text { Oportuni- } \\
\text { dad Desa- } \\
\text { rrollo Pro- } \\
\text { fesional } \\
1.2(\mathrm{~g})\end{array}$ & $\begin{array}{l}\text { Recono- } \\
\text { cimiento } \\
\text { Logros } 1.2 \\
\text { (h) }\end{array}$ & $\begin{array}{l}\text { Oportuni- } \\
\text { dad Ideas } \\
1.2 \text { (i) }\end{array}$ & $\begin{array}{l}\text { Acceso } \\
\text { Estudios } \\
\text { Univer- } \\
\text { sitarios } \\
1.2(\text { (j) }\end{array}$ & $\begin{array}{l}\text { Proceso } \\
\text { de Pla- } \\
\text { nificación } \\
1.2(k)\end{array}$ \\
\hline & $\begin{array}{l}\text { Sig. (bi- } \\
\text { lateral) }\end{array}$ & & & & & & & & & & ,000 & ,000, \\
\hline & $\mathrm{N}$ & & & & & & & & & 439 & 406 & 429 \\
\hline $\begin{array}{l}\text { Acceso } \\
\text { Estudios } \\
\text { Universi- } \\
\text { tarios } 1.2 \\
\text { (j) }\end{array}$ & $\begin{array}{l}\text { Correl- } \\
\text { ación } \\
\text { d e } \\
\text { Pear- } \\
\text { son }\end{array}$ & & & & & & & & & & 1 & ,408(**) \\
\hline & $\begin{array}{l}\text { Sig. (bi- } \\
\text { lateral) }\end{array}$ & & & & & & & & & & & ,000, \\
\hline & $\mathrm{N}$ & & & & & & & & & & 409 & 402 \\
\hline $\begin{array}{l}\text { Proceso } \\
\text { de Planifi- } \\
\text { cación } 1.2 \\
\text { (k) }\end{array}$ & $\begin{array}{l}\text { Correl- } \\
\text { ación } \\
\text { d e } \\
\text { Pear- } \\
\text { son }\end{array}$ & & & & & & & & & & & 1 \\
\hline & $\begin{array}{l}\text { Sig. (bi- } \\
\text { lateral) }\end{array}$ & & & & & & & & & & & \\
\hline & $\mathrm{N}$ & & & & & & & & & & & 437 \\
\hline
\end{tabular}

Tabla 7. Componentes Principales (Recursos v Desarrollo)

Total Variance Explained

\begin{tabular}{|l|r|r|r|r|r|r|}
\hline \multirow{2}{*}{ Component } & \multicolumn{3}{|c|}{ Initial Eigenvalues } & \multicolumn{3}{|c|}{ Extraction Sums of Squared Loadings } \\
\cline { 2 - 7 } & \multicolumn{1}{|c|}{ Total } & \% of Variance & Cumulative \% & \multicolumn{1}{c|}{ Total } & \% of Variance & Cumulative \% \\
\hline 1 & 5,832 & 53,018 & 53,018 & 5,832 & 53,018 & 53,018 \\
2 &, 933 & 8,482 & 61,501 &, 933 & 8,482 & 61,501 \\
3 &, 827 & 7,520 & 69,021 & & & \\
4 &, 564 & 5,130 & 74,151 & & & \\
5 &, 495 & 4,499 & 78,649 & & & \\
6 &, 458 & 4,167 & 82,816 & & & \\
7 &, 433 & 3,938 & 86,754 & & & \\
8 &, 416 & 3,778 & 90,532 & & & \\
9 &, 399 & 3,626 & 94,158 & & & \\
10 &, 359 & 3,264 & 97,423 & & & \\
11 &, 284 & 2,577 & 100,000 & & & \\
\hline
\end{tabular}

Extraction Method: Principal Component Analysis. 
Tabla 8. Pesos de componentes principales (Recursos y Desarrollo)

\begin{tabular}{|c|c|c|}
\hline \multicolumn{3}{|c|}{ Component Matrix } \\
\hline & \multicolumn{2}{|c|}{ Component } \\
\hline & 1 & 2 \\
\hline Recursos Tecnológicos 1.2 (a) & 688 &,- 412 \\
\hline Recursos Mejoramiento Profesional 1.2 (b) & ,773 &,- 262 \\
\hline Recursos Educación Continua 1.2 (c) & 763 & -172 \\
\hline Recursos Financieros 1.2 (d) & 704 & -139 \\
\hline Equipo Disponible 1.2 (e) & ,693 &,- 258 \\
\hline La Inmediatez de Recursos 1.2 (f) & ,778 &,- 116 \\
\hline Oportunidad Desarrollo Prof esional $1.2(\mathrm{~g})$ & ,756 & ,229 \\
\hline Reconocimiento Logros $1.2(\mathrm{~h})$ & ,699 & ,397 \\
\hline Oportunddad Ideas 1.2 (i) &, 750 & ,253 \\
\hline Acceso Estudios Universitarios 1.2 (j) & ,607 &, 540 \\
\hline Proceso de Planificación $1.2(\mathrm{k})$ & ,778 & \\
\hline
\end{tabular}

\section{Análisis de Componentes Prin- cipales: Recursos y Desarrollo}

La matriz de correlaciones de las once variables que intervienen en este apartado se muestra en la tabla 6. Se observa otra vez que todas las correlaciones son positivas y muy significativas.

En este caso el número de variables es mayor y tiene incluso un análisis de componentes principales más sencillo. Los test de Barlett (pvalor igual a 0.0000) y la prueba de idoneidad de Kaiser-Meyer-Olkin (0.934) indican la enorme dependencia entre estas once variables.

El primer componente principal tiene un valor propio igual a 5.83 .

Con dos componentes se explica el $61 \%$ de la variabilidad total de las once variables. (Ver tabla 7). El primer componente es una combinación de las 11 variables, todas con peso positivo. La menos importante corresponde con el Acceso a los estudios Universitarios con peso 0.607 y la más importante la Inmediatez de Recursos con peso 0.778 . (Tabla 8).

\section{Ambiente institucional}

Para evaluar el área ambiente institucional, se utilizaron las preguntas 1.3(a) hasta la $1.3(\mathrm{k})$ del cuestionario. Lo que se pretendía con estas preguntas era evaluar de que forma el cuerpo policiaco estaba satisfecho con el ambiente institucional en la organización. La tasa de respuesta en esta área fue de un $89 \%$. Estas preguntas se diseñaron con una escala de valores que se distribuye entre muy satisfecho (valor 4) hasta muy insatisfecho (valor 1). Como se observa en la tabla 9 y figura 2, la satisfacción promedio en lo que respecta al ambiente institucional en la organización fue de 2,59. Esto demuestra que la mayoría de los encuestados están satisfechos con esta área aunque el promedio se encuentra muy cercano a la media.

Esta área se divide en tres renglones principales, lo que respecta a la reputación del lugar de trabajo, la aportación que realiza la organización a la sociedad y finalmente, los conocimientos y roles del supervisor. Sin embargo, su mayor insatisfacción corresponde a la aportación que hace la agencia a la región y a la sociedad. Estos dos renglones son de vital importancia para este cuerpo, ya que pueden estar observado que su trabajo no es valioso para la sociedad y pueda estar causando efectos negativos en sus ejecutorias.

No obstante, esta insatisfacción con su aportación a la sociedad está relacionada con el área de transparencia de la misión y visión de la Institución, ya que si desconocen el objetivo principal de su organización, puede reflejar una insatisfacción con la aportación que están realizando en la calidad de vida de la sociedad.

El grado de satisfacción en este grupo de preguntas es intermedio entre las preguntas relacionadas con Autoridad y Comunicación y Recursos y Desarrollo. En la tabla 9 se muestran los valores superiores al nivel medio, en el que la respuesta global es de Satisfacción. Otra vez se aprecia que la valoración desciende en las preguntas relativas a la Agencia, como institución externa a la unidad: Reputación Agencia 1.3 (a), Aportación Agencia Región 1.3 (c) y Aportación Agencia Sociedad1.3 (d). La valoración más alta corresponde a la Reputación de la Unidad 1.3 (b) y a las Expectativas Relacionadas con su Supervisor $1.3(\mathrm{k})$. Retornan las diferencias entre los atributos asociados a la unidad y los asociados a la Agencia como institución externa. 
Tabla 9. Valor Medio, Desviación Estándar y Número de Respuestas Ambiente Institucional

\begin{tabular}{|c|c|c|c|}
\hline & Mean & SD & $\mathbf{N}$ \\
\hline Reputación Agencia 1.3 (a) & 2,24 & ,792 & 399 \\
\hline Reputación Unidad 1.3 (b) & $\underline{2,84}$ & ,920 & 399 \\
\hline Aportación Agencia Región 1.3 (c) & 2,20 & ,822 & 399 \\
\hline Aportación Agencia Sociedad 1.3 (d) & 2,44 & ,793 & 399 \\
\hline Conocimiento Supervisor 1.3 (e) & 2,68 & 867 & 399 \\
\hline Reconocimiento Recibido 1.3 (f) & 2,57 & 891 & 399 \\
\hline Supervisor Facilitador 1.3 (g) & 2,62 & ,862 & 399 \\
\hline Evaluación Rendimiento $1.3(\mathrm{~h})$ & 2,79 & 877 & 399 \\
\hline $\begin{array}{l}\text { Conocimiento Profesional Supervisor } \\
1.2(\text { i) }\end{array}$ & 2,74 & ,824 & 399 \\
\hline Necesidades como Empleado 1.2(j) & 2,61 & 867 & 399 \\
\hline $\begin{array}{l}\text { Expectativas Supervisor Resultados } \\
1.2(\mathrm{k})\end{array}$ & 2,68 & ,822 & 399 \\
\hline
\end{tabular}

Tabla 10. Matriz de Correlaciones: Ambiente Institucional

\begin{tabular}{|c|c|c|c|c|c|c|c|c|c|c|c|c|}
\hline & & $\begin{array}{c}\text { Repu- } \\
\text { t a c i ó } n \\
\text { Agencia } \\
1.3 \text { (a) }\end{array}$ & $\begin{array}{c}\text { Repu- } \\
\text { t a ción } \\
\text { Unida d } \\
1.3 \text { (b) }\end{array}$ & $\begin{array}{c}\text { A por } \\
\text { tación } \\
\text { Agencia } \\
\text { Región } \\
1.3 \text { (c) }\end{array}$ & $\begin{array}{c}\text { Apor- } \\
\text { t a c i ó n } \\
\text { Agencia } \\
\text { Sociedad } \\
1.3 \text { (d) }\end{array}$ & $\begin{array}{l}\text { Conoci- } \\
\text { miento } \\
\text { Supervi- } \\
\text { sor } 1.3 \\
\text { (e) }\end{array}$ & $\begin{array}{c}\text { Recono- } \\
\text { cimiento } \\
\text { Recibido } \\
1.3 \text { (f) }\end{array}$ & $\begin{array}{l}\text { Supervi- } \\
\text { sor Faci- } \\
\text { lit a d o } r \\
1.3(\mathrm{~g})\end{array}$ & $\begin{array}{l}\text { Eva- } \\
\text { luación } \\
\mathrm{R} \text { e } \mathrm{n} \text { - } \\
\text { dimien- } \\
\text { to } 1.2 \\
\text { (h) }\end{array}$ & $\begin{array}{l}\text { Conoci- } \\
\text { miento } \\
\text { Profe- } \\
\text { sional } \\
\text { Sup. } 1.2 \\
\text { (i) }\end{array}$ & $\begin{array}{c}\text { Necesi- } \\
\text { dades } \\
\text { c o m o } \\
\text { E m - } \\
\text { pleado } \\
1.2(j)\end{array}$ & $\begin{array}{l}\text { Expec- } \\
\text { tativas } \\
\text { Supervi- } \\
\text { sor Re- } \\
\text { sultados } \\
1.2(k)\end{array}$ \\
\hline \multirow[t]{3}{*}{$\begin{array}{l}\text { Reputación } \\
\text { Agencia } 1.3 \\
\text { (a) }\end{array}$} & $\begin{array}{l}\text { Correl- } \\
\text { ación de } \\
\text { Pearson }\end{array}$ & 1 &, $\left.393^{(* *}\right)$ &, $\left.450^{(* *}\right)$ &, $522\left(^{* *}\right)$ &, $4011^{(*)}$ &, $\left.392^{(* *}\right)$ &, $\left.383^{(* *}\right)$ &, $\left.340^{(* *}\right)$ &, $\left.383^{(* *}\right)$ &, $\left.375^{(* *}\right)$ &, $318\left(^{* \star}\right)$ \\
\hline & $\begin{array}{l}\text { Sig. (bi- } \\
\text { lateral) }\end{array}$ & & ,000 & ,000 & ,000 & ,000 & ,000 & ,000 & 000 & ,000 & ,000 & 000 \\
\hline & $\mathrm{N}$ & 441 & 439 & 432 & 435 & 436 & 432 & 435 & 433 & 433 & 434 & 424 \\
\hline \multirow[t]{3}{*}{$\begin{array}{l}\text { Reputación } \\
\text { Unidad } 1.3 \\
\text { (b) }\end{array}$} & $\begin{array}{l}\text { Correl- } \\
\text { ación de } \\
\text { Pearson }\end{array}$ & & 1 &, $333\left(^{(* *)}\right.$ &, $303^{\left({ }^{*}\right)}$ &, $377\left(^{(* *)}\right.$ &, $\left.4011^{(\star *}\right)$ &, $410{ }^{\left({ }^{*}\right)}$ &, $377\left(^{(*)}\right)$ &, $400{ }^{\left({ }^{*}\right)}$ &, $373\left(^{\left({ }^{\star *}\right)}\right.$ &, $479\left(^{* \star}\right)$ \\
\hline & $\begin{array}{l}\text { Sig. (bi- } \\
\text { lateral) }\end{array}$ & & & ,000 & ,000 & ,000 & ,000 & ,000 & ,000 & ,000 & ,000 & 000 \\
\hline & $\mathrm{N}$ & & 445 & 434 & 439 & 440 & 435 & 438 & 436 & 437 & 438 & 428 \\
\hline
\end{tabular}




\begin{tabular}{|c|c|c|c|c|c|c|c|c|c|c|c|c|}
\hline & & $\begin{array}{c}\text { Repu- } \\
\text { t a c i ó n } \\
\text { Agencia } \\
1.3 \text { (a) }\end{array}$ & $\begin{array}{c}\text { Repu- } \\
\text { t a c ió n } \\
\text { Unidad } \\
1.3 \text { (b) }\end{array}$ & $\begin{array}{c}\text { A por - } \\
\text { tación } \\
\text { Agencia } \\
\text { Región } \\
1.3 \text { (c) }\end{array}$ & $\begin{array}{c}\text { Apor- } \\
\text { t a c i ón } \\
\text { Agencia } \\
\text { Sociedad } \\
1.3 \text { (d) }\end{array}$ & $\begin{array}{l}\text { Conoci- } \\
\text { miento } \\
\text { Supervi- } \\
\text { sor } 1.3 \\
\text { (e) }\end{array}$ & $\begin{array}{c}\text { Recono- } \\
\text { cimiento } \\
\text { Recibido } \\
1.3 \text { (f) }\end{array}$ & $\begin{array}{l}\text { Supervi- } \\
\text { sor Faci- } \\
\text { lit a d o r } \\
1.3(\mathrm{~g})\end{array}$ & $\begin{array}{l}\text { Eva- } \\
\text { luación } \\
\mathrm{R} \text { e } \mathrm{n} \text { - } \\
\text { dimien- } \\
\text { to } 1.2 \\
\text { (h) }\end{array}$ & $\begin{array}{l}\text { Conoci- } \\
\text { miento } \\
\text { Profe- } \\
\text { sional } \\
\text { Sup. } 1.2 \\
\text { (i) }\end{array}$ & $\begin{array}{l}\text { Necesi- } \\
\text { dades } \\
\text { co mo } \\
\text { E m - } \\
\text { pleado } \\
1.2(j)\end{array}$ & $\begin{array}{c}\text { Expec- } \\
\text { tativas } \\
\text { Supervi- } \\
\text { sor Re- } \\
\text { sultados } \\
1.2(k)\end{array}$ \\
\hline \multirow[t]{3}{*}{$\begin{array}{l}\text { Aportación } \\
\text { A g e n cia } \\
\text { Región } 1.3 \\
\text { (c) }\end{array}$} & $\begin{array}{l}\text { Correl- } \\
\text { ación de } \\
\text { Pearson }\end{array}$ & & & 1 &, $466\left(^{* \star}\right)$ &, $337\left(^{* \star}\right)$ &, $354\left(^{\left({ }^{\star *}\right)}\right.$ &, $\left.370^{(* *}\right)$ &, $336\left(^{\star \star *}\right)$ &, $317\left(^{(* *}\right)$ &, $\left.2599^{(* *}\right)$ &, $293\left({ }^{(\star *}\right)$ \\
\hline & $\begin{array}{l}\text { Sig. (bi- } \\
\text { lateral) }\end{array}$ & & & & , 000 & ,000 & ,000 & ,000 & ,000 & ,000 & ,000 & ,000 \\
\hline & $\mathrm{N}$ & & & 436 & 433 & 431 & 428 & 430 & 426 & 429 & 430 & 421 \\
\hline \multirow[t]{3}{*}{$\begin{array}{l}\text { Aportación } \\
\text { Agen cia } \\
\text { Sociedad } \\
1.3 \text { (d) }\end{array}$} & $\begin{array}{l}\text { Correl- } \\
\text { ación de } \\
\text { Pearson }\end{array}$ & & & & 1 &, $\left.319^{(* \star}\right)$ &, $\left.355^{(* \star}\right)$ &, $\left.292{ }^{* \star}\right)$ &, $278\left(^{(\star *}\right)$ &, $\left.311{ }^{* \star}\right)$ &, $\left.290{ }^{* *}\right)$ &, $247\left(^{* \star}\right)$ \\
\hline & $\begin{array}{l}\text { Sig. (bi- } \\
\text { lateral) }\end{array}$ & & & & & ,000 & ,000 & ,000 & ,000 & ,000 & ,000 & ,000 \\
\hline & $\mathrm{N}$ & & & & 441 & 435 & 433 & 435 & 431 & 434 & 435 & 426 \\
\hline \multirow[t]{3}{*}{$\begin{array}{l}\text { Conoci- } \\
\text { miento Su- } \\
\text { pervisor } 1.3 \\
\text { (e) }\end{array}$} & $\begin{array}{l}\text { Correl- } \\
\text { ación de } \\
\text { Pearson }\end{array}$ & & & & & 1 &, $688\left(^{\left({ }^{\star *}\right)}\right.$ &, $\left.700^{(* *}\right)$ &, $575\left(^{(\star *}\right)$ &, $\left.782^{(* *}\right)$ &, $\left.6622^{(* *}\right)$ &, $6422^{\left({ }^{\star *}\right)}$ \\
\hline & $\begin{array}{l}\text { Sig. (bi- } \\
\text { lateral) }\end{array}$ & & & & & & ,000 & ,000 & ,000 & ,000 & ,000 & ,000 \\
\hline & $\mathrm{N}$ & & & & & 442 & 433 & 437 & 434 & 437 & 437 & 426 \\
\hline \multirow[t]{3}{*}{$\begin{array}{l}\text { Reconoci- } \\
\text { miento Re- } \\
\text { cibido } 1.3 \text { (f) }\end{array}$} & $\begin{array}{l}\text { Correl- } \\
\text { ación de } \\
\text { Pearson }\end{array}$ & & & & & & 1 &, $\left.744^{(* *}\right)$ &, $638\left(^{\left({ }^{* *}\right)}\right.$ &, $\left.6544^{(\star *}\right)$ &, $\left.6722^{* \star}\right)$ &, $657\left(^{\left({ }^{\star *}\right)}\right.$ \\
\hline & $\begin{array}{l}\text { Sig. (bi- } \\
\text { lateral) }\end{array}$ & & & & & & & ,000 & ,000 & ,000 & ,000 & ,000 \\
\hline & $\mathrm{N}$ & & & & & & 437 & 433 & 430 & 432 & 433 & 423 \\
\hline \multirow[t]{3}{*}{$\begin{array}{l}\text { Supervisor } \\
\text { Facilitador } \\
1.3(\mathrm{~g})\end{array}$} & $\begin{array}{l}\text { Correl- } \\
\text { ación de } \\
\text { Pearson }\end{array}$ & & & & & & & 1 &, $648\left(^{\left({ }^{\star *}\right)}\right.$ & ,694(**) &, $703\left(^{(* *}\right)$ &, $647\left(^{* \star}\right)$ \\
\hline & $\begin{array}{l}\text { Sig. (bi- } \\
\text { lateral) }\end{array}$ & & & & & & & & ,000 & ,000 & ,000 & ,000 \\
\hline & $\mathrm{N}$ & & & & & & & 439 & 433 & 436 & 437 & 426 \\
\hline
\end{tabular}




\begin{tabular}{|c|c|c|c|c|c|c|c|c|c|c|c|c|}
\hline & & $\begin{array}{c}\text { Repu- } \\
\text { t a c i ó n } \\
\text { Agencia } \\
1.3 \text { (a) }\end{array}$ & $\begin{array}{c}\text { Repu- } \\
\text { t a c i ó n } \\
\text { Unida d } \\
1.3 \text { (b) }\end{array}$ & $\begin{array}{c}\text { A por - } \\
\text { tación } \\
\text { Agencia } \\
\text { Región } \\
1.3 \text { (c) }\end{array}$ & $\begin{array}{c}\text { Apor- } \\
\text { t a c ión } n \\
\text { Agencia } \\
\text { Sociedad } \\
1.3 \text { (d) }\end{array}$ & $\begin{array}{l}\text { Conoci- } \\
\text { miento } \\
\text { Supervi- } \\
\text { sor } 1.3 \\
\text { (e) }\end{array}$ & $\begin{array}{c}\text { Recono- } \\
\text { cimiento } \\
\text { Recibido } \\
1.3 \text { (f) }\end{array}$ & $\begin{array}{l}\text { Supervi- } \\
\text { sor Faci- } \\
\text { lit a d or } \\
1.3(\mathrm{~g})\end{array}$ & $\begin{array}{l}\text { Eva- } \\
\text { luación } \\
\mathrm{R} \text { e } \mathrm{n} \text { - } \\
\text { dimien- } \\
\text { to } 1.2 \\
\text { (h) }\end{array}$ & $\begin{array}{l}\text { Conoci- } \\
\text { miento } \\
\text { Profe- } \\
\text { sional } \\
\text { Sup. } 1.2 \\
\text { (i) }\end{array}$ & $\begin{array}{l}\text { Necesi- } \\
\text { dades } \\
\text { c o mo } \\
\text { E m - } \\
\text { pleado } \\
1.2(j)\end{array}$ & $\begin{array}{c}\text { Expec- } \\
\text { tativas } \\
\text { Supervi- } \\
\text { sor Re- } \\
\text { sultados } \\
1.2(k)\end{array}$ \\
\hline $\begin{array}{l}\text { Evaluación } \\
\text { Rendimien- } \\
\text { to } 1.2(\mathrm{~h})\end{array}$ & $\begin{array}{l}\text { Correl- } \\
\text { ación de } \\
\text { Pearson }\end{array}$ & & & & & & & & 1 & ,621 $\left.1^{\star *}\right)$ & ,620(**) &, $616\left({ }^{\star \star}\right)$ \\
\hline & $\begin{array}{l}\text { Sig. (bi- } \\
\text { lateral) }\end{array}$ & & & & & & & & & ,000 & ,000 & ,000 \\
\hline & $\mathrm{N}$ & & & & & & & & 437 & 431 & 432 & 423 \\
\hline $\begin{array}{l}\text { Conoci- } \\
\text { m i e n t o } \\
\text { Profesional } \\
\text { Sup. } 1.2 \text { (i) }\end{array}$ & $\begin{array}{l}\text { Correl- } \\
\text { ación de } \\
\text { Pearson }\end{array}$ & & & & & & & & & 1 &, $725^{\left({ }^{\star *}\right)}$ &, $672\left(^{(* *}\right)$ \\
\hline & $\begin{array}{l}\text { Sig. (bi- } \\
\text { lateral) }\end{array}$ & & & & & & & & & & ,000 & ,000 \\
\hline & $\mathrm{N}$ & & & & & & & & & 439 & 437 & 427 \\
\hline $\begin{array}{l}\mathrm{Nec} \text { e } \mathrm{s} \text { i - } \\
\text { dades como } \\
\text { Empleado } \\
1.2(\mathrm{j})\end{array}$ & $\begin{array}{l}\text { Correl- } \\
\text { ación de } \\
\text { Pearson }\end{array}$ & & & & & & & & & & 1 & ,689(**) \\
\hline & $\begin{array}{l}\text { Sig. (bi- } \\
\text { lateral) }\end{array}$ & & & & & & & & & & & ,000 \\
\hline & $\mathrm{N}$ & & & & & & & & & & 439 & 427 \\
\hline $\begin{array}{l}\text { Expecta- } \\
\text { tivas Su- } \\
\text { pervisor } \\
\text { Resultados } \\
1.2(k)\end{array}$ & $\begin{array}{l}\text { Correl- } \\
\text { ación de } \\
\text { Pearson }\end{array}$ & & & & & & & & & & & 1 \\
\hline & $\begin{array}{l}\text { Sig. (bi- } \\
\text { lateral) }\end{array}$ & & & & & & & & & & & \\
\hline & $\mathrm{N}$ & & & & & & & & & & & 429 \\
\hline
\end{tabular}


Tabla 11. Análisis de Componentes Principales Ambiente Institucional

Total Variance Explained

\begin{tabular}{|l|r|r|r|r|r|r|}
\hline \multirow{2}{*}{ Component } & \multicolumn{3}{|c|}{ Initial Eigenvalues } & \multicolumn{3}{|c|}{ Extraction Sums of Squared Loadings } \\
\cline { 2 - 7 } & \multicolumn{1}{|c|}{ Total } & \% of Variance & Cumulative \% & Total & \% of Variance & Cumulativ e $\%$ \\
\hline 1 & 6,028 & 54,800 & 54,800 & 6,028 & 54,800 & 54,800 \\
2 & 1,372 & 12,477 & 67,277 & 1,372 & 12,477 & 67,277 \\
3 &, 712 & 6,475 & 73,752 & & & \\
4 &, 584 & 5,305 & 79,056 & & & \\
5 &, 466 & 4,240 & 83,296 & & & \\
6 &, 421 & 3,824 & 87,121 & & & \\
7 &, 372 & 3,382 & 90,502 & & & \\
8 &, 344 & 3,125 & 93,628 & & & \\
9 &, 303 & 2,759 & 96,386 & & & \\
10 &, 220 & 1,998 & 98,384 & & & \\
11 &, 178 & 1,616 & 100,000 & & & \\
\hline
\end{tabular}

Extraction Method: Principal Component Analysis.

Tabla 12. Pesos de Componentes Principales: Ambiente Institucional

Component Matrix
\begin{tabular}{|l|r|r|}
\hline \multicolumn{2}{|c|}{ Component } \\
\cline { 2 - 3 } Reputación Agencia 1.3 (a) & 1 & \multicolumn{1}{c|}{2} \\
Reputación Unidad 1.3 (b) &, 578 &, 558 \\
Aportación Agencia Región 1.3 (c) &, 595 &, 200 \\
Aport ación Agencia Sociedad 1.3 (d) &, 515 &, 590 \\
Conocimiento Superv isor 1.3 (e) &, 499 &, 649 \\
Reconocimiento Recibido 1.3 (f) &, 843 &,- 164 \\
Superv isor Facilitador 1.3 (g) &, 843 &,- 120 \\
Ev aluación Rendimiento 1.2 (h) &, 851 &,- 176 \\
Conocimiento Profesional Sup. 1.2 (i) &, 789 &,- 147 \\
Necesidades como Empleado 1.2 (j) &, 849 &,- 198 \\
Expectativas Supervisor Resultados 1.2 (k) &, 825 &,- 249 \\
Extraction Method: Principal Component Analy sis. &, 810 &,- 236 \\
\hline
\end{tabular} a. 2 components extracted.

\section{Análisis de Componentes Prin- cipales: Ambiente Institucional}

El indicador de satisfacción relativo al grupo de preguntas del epígrafe de Ambiente Institucional. Como se aprecia la matriz de correlaciones presenta las mismas propiedades que en los dos casos anteriores. Algunos coeficientes son especialmente altos, por ejemplo entre Reconocimiento Recibido y Supervisor Facilitador.

Estos aspectos deben ser valorados muy especialmente en organizaciones como la policiaca donde existe una jerarquía muy fuerte y las reglas de funcionamiento se basan fundamentalmente en seguir las órdenes del superior. La satisfacción personal del policía está muy relacionada con aspectos que dependen de la comunicación, reconocimientos de méritos y relaciones con sus superiores. El contraste de Bartlett ( $p$ valor 0.000 ) y la medida de adecuación muestra de Kaiser-Mayer-Olkin (0.931) revelan la enorme dependencia existente entre estas once variables. La solución de dos componentes principales se muestra a continuación.

Destacamos la gran importancia del primer componente (tabla 11), con un valor propio superior a 6 , explica él solo más del $50 \%$ de la varianza de las once variables.

Esto es especialmente relevante en nuestro análisis basado en un único indicador. El segundo compo- 
nente tiene un valor propio superior a 1 . Entre los dos factores se explica el $67 \%$ de la varianza total.

Los pesos del primer componente (tabla 12) muestra la diferencia de la importancia de los cuatro primeros ítems (relativos a la agencia) y el resto de las preguntas (relativas a la unidad).

\section{Comparación de la satisfac- ción por grupos}

La figura 1 muestra gráficamente los valores medios recogidos en las tablas superiores. La línea roja marca el nivel de 2.5. Lo más destacado de la figura es que las valoraciones relativas al desarrollo profesional se encuentran muy por debajo del nivel 2.5 y es muy inferior a la valoración de los restantes conceptos.

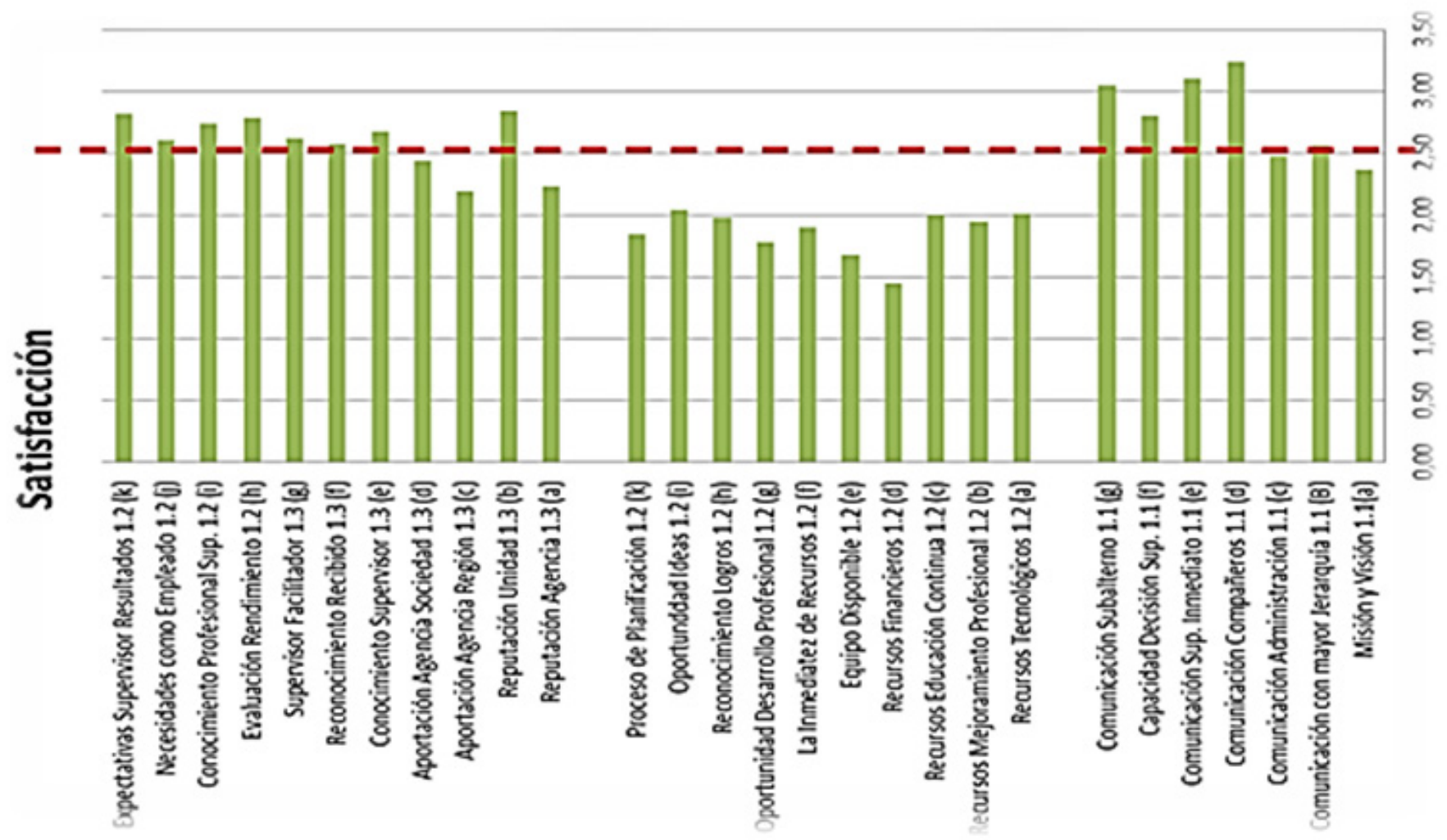

Figura 2. Comparación de la satisfacción por grupos.

\section{Conclusión}

La satisfacción laboral sin duda es un factor conducente a un mejor desempeño. Sin embargo el pasar por desapercibido la importancia de la satisfacción laboral puede atraer consigo serias dificultades en una organización mayor aún cuyo recurso principal es el ser humano. En el renglón de autoridad y comunicación las correlaciones se identifican como altas y positivas entre todas las variables, además todas son significativas. El análisis realizado muestra que el aspecto que más valoran los policías entre la Autoridad y Comunicación es la comunicación con los superiores siendo el superior inmediato el que mayor peso otorga.

En el ámbito de Recursos y Desarrollo la Insatisfacción se describe con mayor evidencia en los aspectos relacionados con los recursos financieros, el equipo disponible y oportunidades para el desarrollo profesional.

En el ambiente institucional se aprecia que la valoración de insatisfacción desciende en las preguntas relativas a la Agencia, como institución externa a la unidad: Reputación Agencia, Aportación Agencia a la Región y Aportación Agencia a la Sociedad. 
Sin embargo el policía valora la reputación de la Unidad en donde trabaja y las expectativas relacionadas con su supervisor inmediato. Estos aspectos deben ser valorados muy especialmente en organizaciones como la policiaca donde existe una jerarquía muy fuerte y las reglas de funcionamiento se basan fundamentalmente en seguir las órdenes del superior.

La satisfacción personal del policía está muy relacionada con aspectos que dependen de la comunicación, reconocimientos de méritos y relaciones con sus superiores muy en especial hacia los rangos de sargento. Se aprecia en la ecuación que las variables que influyen de manera más significativa en la satisfacción laboral son los conocimientos y aptitudes del Supervisor $1.3(\mathrm{e})$, reconocimiento recibido por parte del Supervisor 1.3 (f), el rol del Supervisor como facilitador $1.3(\mathrm{~g})$, la evaluación del Supervisor hacia el rendimiento profesional $1.2(\mathrm{~h})$, con los conocimientos profesionales del Supervisor 1.2 (i), la información que posee el Supervisor sobre necesidades como empleado 1.2 (j) y las expectativas del Supervisor sobre los resultados $1.2(\mathrm{k})$.

\section{Recomendaciones}

La comprensión de cómo reclutar, entrenar y mantener una fuerza policial eficaz es un objetivo importante tal como lo define (Egan, 2005) pero es transcendental que la organización policiaca desarrolle el tiempo y energías necesarias para colonizar y fortalecer el recurso que se inserta día a día en las tareas policiales.

Tal como lo establece (Miranda, 2009) la policía debe tener como principios un servicio civil encaminado a la preservación de la seguridad y de la justicia, la protección de los ciudadanos, la protección de los derechos humanos, de la vida política democrática, que se conduce con apego a la transparencia y la rendición de cuentas todas y cada una de ellas dependiente de la cultura organizacional de la institución policial.

La Policía ha de compatibilizar un paradigma racional burocrático con la necesidad de responder a lo imprevisto de una manera ágil (Torrente, 1992). No obstante es contingente el obedecer a una administración del recurso humano proactiva y dotar al policía de una valorización de lo que manifiesta satisfacción muy relacionada en nuestros hallazgos con actitudes personales del policía, incursionado en el (desempeño) y fuera de su puesto de trabajo.

\section{Referencias}

ÁLVAREZ, J. (2009). Estilos de Liderazgo en la Policía Local de la Comunidad Valenciana. Universidad de Valencia. Facultad de Psicología.

BAYLEY, D. H. (2001). Democratizing the Police Abroad: What to do and How to do it. USA. National Institute of Justice.

BITTNER, E. (1970). The functions of police in modern society. Rockville, Md: National Institute of Mental Health.

BITTNER, E. (1990). Aspects of police work. Boston: Northeastern University Press.

BRUCE, D. (2003). Democratic reform of police any lessons for Kenya from South Africa? Centre for the Study of Violence and Reconciliation, Johannesburg.

CHERNISS, C. (1980). Staff burnout: Job stress in the human services. Beverly Hills: Sage.

CHIANG, V.M., SALAZAR, B.C. and NUÑEZ, P.A. (2007). "Clima organizacional y satisfacción laboral en un establecimiento de salud mental: Hospital Tipo I". Theoría, 16 (2), PP. 61-76.

DOHERTY, T. L. and T. HORNE. (2001). Managing Public Services-Implementing Changes: A Thoughtful Approach to the Practice of Management. NY: Taylor and Francis Group.

EGAN, T. (2005). "Police forces, their ranks thin, offer bonuses, bounties and more". New York Times, 155: page A1 December 28, 2005.

FOGELSON, R.M. (1977). Big-city police. Cambridge: Harvard University Press.

FROST, J. (2006). "Predictors of Job Satisfaction and Turnover Intention In Police Organizations: A procedural Approach. Criminal Justice Graduate College of the University of Illinois at Chicago". Future Research. Police Chief. 64, pp. 97-99.

GABRIUNAS, I. P. (2010). "La Influencia del Desempeño Social Corporativo en la Satisfacción Laboral de los Empleados: Una revisión Teórica desde una Perspectiva Multinivel". Estudios Gerenciales, 26(116), pp. 63-81. Retrieved from http://search.proquest. com/docview/818327959? accountid=28867 
GOLTZ, J. (2006). Police Organizational Performance in the State of Florida: Confirmatory Analysis of the Relationship of the environment and Design Structure to Performance. College of Health and Public Affairs at the University of Central Florida Orlando, Florida.

GORIS, J. R., VAUGHT, B. C., and PETTIT., J. D. (2000). "Effects of communication direction on job performance and satisfaction: A moderated regression analysis". Journal of Business Communication,37 (4), pp. 348-368.

GRIFFIN, G.R., DUNBAR, R.L.M., and MCGRILL, M.E. (1978). "Factors associated with job satisfaction among police personnel". Journal of Police Science and Administration, 5 (1), pp. 77-85.

HARRISON, D.A., NEWMAN, D.A. and ROTH, P.L. (2006). "How important are job attitudes? Meta- analytic comparisons of integrative behavioral outcomes and time sequences". Academy of Management Journal, 49(2), pp. 305-325.

KROES, W.H. (1985). Society's victims-The police: 2nd ed. Springfield, II:

THOMAS, C. (1986). Society's victim: The police officer. Springfield, II:

THOMAS, C. (1976). Society's victim, the policeman: An analysis of job stress in policing. New York: Charles C. Thomas.

MANNING, P.K. (1977). The police: Mandate, strategies and appearances. In J.D.

MELÉNDEZ, O. (2013). Director Regional. Comandancia de la Policía de Puerto Rico, Región de Humacao. Policía de Puerto Rico.

MIRANDA, C. (2009). Los Retos de la Reforma Policial y la Capacitación en el Marco del Estado de Derecho en México. Facultad Latinoamericana de Ciencias Sociales, México Maestría en Derechos Humanos y Democracia. FLACSO México.

MIRE, S. (2007). Correlates of Job Satisfaction Among Police Officers. The Faculty of the College of Criminal Justice. Sam Houston State University. UMI Number: 3190117

MONSALVE, J. N. M., BRONCANO, S. G., and NIÑO, A. M. (2012). "Las Prácticas de alto rendimiento influencia de la autoridad no formal en la administración del talento humano y su impacto en los re- sultados empresariales". Paper presented at the , 7(1) 1322-1334. Retrieved from http://search.proquest.com/docview $/ 1323545960$ ?accountid=28867

PATLÁN, J., MARTÍNEZ, E,.and HERNÁNDEZ, R. (2012). "El Clima y la Justicia Organizacional y su Efecto en la Satisfacción Laboral". Revista Internacional Administración \& Finanzas, 5, (5), 2012.

POPOVICH, M. G. (1998). Creating High-Performance Government Organizations. San Francisco, CA: Jossey-Bass Inc.

SEVEN, H. (2012). An Analysis of the Effect of Internal Communication Satisfaction on Organizational Commitment in the Turkish National Police (TNP). University of Baltimore. School of Public Affairs.

SHANE, J. M., (2008). Organizational stressors and police performance. Graduate School-Newark Rutgers, The State University of New Jersey

SHANE, J. M. (2010), "Organizational stressors and police performance", Journal of Criminal Justice doi:10.1016/j. jcrimjus.2010.05.008

SILVERMAN, E. B. (1999). NYPD Battles Crime: Innovative Strategies in Policing. MA: Northeastern University Press.

SPECTOR, P. (1985). "Measurement of human service staff satisfaction: development of the Job Satisfaction Survey". American Journal of Community Psychology, 13, pp. 693-713.

TORRENTE, D. (1992). "Investigando a la policía. Revista Española de Investigaciones Sociológicas, 59, pp. 289-300.

TORRENTE, D. (1997). La sociedad policial. Barcelona: CIS.

ULUTURK, B. (2012). An Assessment of Law Enforcement Officers' Attitudes toward Compstat Model of Police Management. University of Baltimore. School of Public Affair. Violanti, J. M., \& Aron, F. (1993). Sources of police stressors, job attitudes and psychological distress. Psychological Reports, 72, 899-904.

WILLIAM J. BRATTON, "Crime is Down in New York City: Blame the Police," Zero Tolerance: Policing a Free Society, enlarged and revised second edition, ed. Norman Dennis (London: IEA Health and Welfare Unit, 1998) 
WILLIAMS, L.J. AND HAZER, J.T. (1986). "Antecedents and consequences of satisfaction and commitment in turnover models: a re-analysis using latent variable structural equation methods". Journal of $A p$ plied Psychology, 72 (1), pp. 219-31.

WILSON, J. Q. (1968). Varieties of Police Behavior. New York: Atheneum.
ZHAO, J., THURMAN, C. and HE, N. (1999). "Sources of job satisfaction among police officers: A test of demographic and work environment models". Justice Quarterly, 16, (1), pp.153-173. 